\title{
BMJ Open Interventions for the prediction and management of chronic postsurgical pain after total knee replacement: systematic review of randomised controlled trials
}

\author{
Andrew D Beswick, Vikki Wylde, Rachael Gooberman-Hill
}

To cite: Beswick $A D$, Wylde V, Gooberman-Hill R. Interventions for the prediction and management of chronic postsurgical pain after total knee replacement: systematic review of randomised controlled trials. BMJ Open 2015;5:e007387. doi:10.1136/bmjopen-2014007387

- Prepublication history and additional material is available. To view please visit the journal (http://dx.doi.org/ 10.1136/bmjopen-2014007387).

Received 9 December 2014 Revised 16 March 2015 Accepted 10 April 2015

CrossMark

Musculoskeletal Research Unit, School of Clinical Sciences, University of Bristol, Bristol, UK

Correspondence to Andrew D Beswick; andy.beswick@bristol.ac.uk

\section{ABSTRACT}

Objectives: Total knee replacement can be a successful operation for pain relief. However, 10-34\% of patients experience chronic postsurgical pain. Our aim was to synthesise evidence on the effectiveness of applying predictive models to guide preventive treatment, and for interventions in the management of chronic pain after total knee replacement.

Setting: We conducted a systematic review of randomised controlled trials using appropriate search strategies in the Cochrane Library, MEDLINE and EMBASE from inception to October 2014. No language restrictions were applied.

Participants: Adult patients receiving total knee replacement.

Interventions: Predictive models to guide treatment for prevention of chronic pain. Interventions for management of chronic pain.

Primary and secondary outcome measures: Reporting of specific outcomes was not an eligibility criterion but we sought outcomes relating to pain severity.

Results: No studies evaluated the effectiveness of predictive models in guiding treatment and improving outcomes after total knee replacement. One study evaluated an intervention for the management of chronic pain. The trial evaluated the use of a botulinum toxin $A$ injection with antinociceptive and anticholinergic activity in 49 patients with chronic postsurgical pain after knee replacement. A single injection provided meaningful pain relief for about 40 days and the authors acknowledged the need for a large trial with repeated injections. No trials of multidisciplinary interventions or individualised treatments were identified.

Conclusions: Our systematic review highlights a lack of evidence about the effectiveness of prediction and management strategies for chronic postsurgical pain after total knee replacement. As a large number of people are affected by chronic pain after total knee replacement, development of an evidence base about care for these patients should be a research priority.

\section{Strengths and limitations of this study}

- Reproducible systematic review methods.

- Identification of research priorities.

- Interventions for chronic pain after other surgeries may have value in total knee replacement.

\section{INTRODUCTION}

Total knee replacement is an increasingly common procedure that aims to reduce pain and functional limitations, particularly for people with osteoarthritis of the knee. In the year to 31 March 2014, nearly 78000 people received a primary total knee replacement in the UK, ${ }^{1}$ and in 2010 approximately 719000 procedures were performed in the USA. ${ }^{2}$ It is estimated that over half of all people in the USA diagnosed with osteoarthritis will receive a total knee replacement. ${ }^{3}$

Surgery is a known risk factor for chronic pain ${ }^{4}$ defined as pain "present for at least 3 months'. ${ }^{5}$ Chronic postsurgical pain 'develops after a surgical procedure or increases in intensity after the surgical procedure'. Although many patients report a good outcome after their total knee replacement, at a time when recovery should have been achieved, ${ }^{7}$ about $10-34 \%$ of patients report moderate to severe chronic postsurgical pain. ${ }^{8}$ In the UK, this could mean 7500-25 500 potential new cases of chronic postsurgical pain every year, while in the USA this equates to between 72000 and a quarter of a million new cases annually. As patients undergo knee replacement in order to relieve knee pain, these estimates are cause for concern.

Given the distress caused by chronic postsurgical pain, ${ }^{9}$ and the predicted increases in prevalence of osteoarthritis, ${ }^{10}$ and the need for knee replacement surgery, ${ }^{11}$ robust 
evidence is needed on effective methods for preventing the development of chronic pain, identifying patients at risk of developing chronic pain, and for the management of chronic pain. Inadequately controlled perioperative pain is a risk factor for long-term pain and, although studied widely, systematic reviews have shown that evidence on long-term benefit is limited. ${ }^{12-15}$

A large number of preoperative and early postoperative factors are associated with poor pain outcomes, including greater joint pain ${ }^{16-18}$ and pain catastrophisation, ${ }^{19-21}$ poor mental health, ${ }^{16} 1921-23$ and presence of musculoskeletal comorbidities. ${ }^{18}{ }^{24}$ As the cause of chronic pain after total knee replacement is likely to be multifactorial, with mechanical, biological and psychological features, simple interventions targeting individual issues will leave a large proportion of patients at risk of developing long-term pain with no appropriate care. The potential value of multivariable risk assessment is clear although the ability of predictive models to identify patients at risk of long-term pain has been highly variable. ${ }^{25}{ }^{26}$ Furthermore, as with all prognostic models guiding decision-making, evidence on their efficacy and safety in targeting interventions is required before application in clinical practice. ${ }^{27}$

Owing to the complexity of chronic pain, treatments in appropriate combinations matched to patient characteristics are advocated. ${ }^{28}{ }^{29}$ As with application of methods for prediction, evidence is required that pain management strategies are effective in patients with chronic postsurgical pain after total knee replacement. This may relate to specific treatments or to multifactorial assessment and management.

Our aim was to conduct a systematic review to identify randomised trials in patients with total knee replacement that have evaluated: (1) the application of predictive models in the targeting of pain management and (2) interventions for the treatment of chronic pain. Relevant outcomes related to pain severity. While our particular interest was total knee replacement, we used a broader search strategy to include any type of knee surgery as appropriate pain prediction, and management methods may have been evaluated in more diverse knee surgeries.

\section{METHODS}

We aimed to conduct our literature reviews with transparent and unbiased methods such that they can be considered truly systematic and reproduced on the basis of sources of literature, search processes, study inclusion or exclusion, data analysis if feasible, and study quality assessment. To achieve this, we used methods described in the Cochrane Handbook of Systematic reviews. ${ }^{30}$ As this review focuses on randomised controlled trials, we conducted the review with reference to Preferred Reporting Items for Systematic Reviews and Meta-Analyses (PRISMA) guidelines which aim to improve the reporting of systematic reviews. ${ }^{31}$

\section{Search strategy}

Separate literature search strategies for predictive methods and pain management based on validated searches $^{30} 3233$ were applied in MEDLINE, EMBASE and the Cochrane Library from inception up to 1 October 2014. We considered that the EMBASE coverage of conference abstracts since 2009 was an appropriate search of 'grey literature' in the orthopaedic context. Search strategies as applied in MEDLINE are shown in box 1 with combinations of terms, such as 'risk function, risk assessment, randomised trial, knee,' and 'pain, postoperative, post-surgical, randomised trial, knee.' Search terms were in English, but no further language restrictions were applied with funds available to pay translation costs if required. If necessary author contact for additional information was planned.

\section{Eligibility criteria}

Eligible studies satisfied PICOS criteria.

- Patients: adults with knee surgery

- Intervention: treatment guided by a predictive model or an intervention for management of chronic pain (pain reported at 3 months or more after surgery)

- Control: a usual care comparison group

- Outcome: an outcome relating to pain severity

- Setting: evaluation in a randomised controlled trial

\section{Data extraction}

Articles and inclusion/exclusion decisions were catalogued in Endnote X7. All titles and abstracts were screened independently by two reviewers. Potentially relevant articles were evaluated in detail by two reviewers, independently, with decisions on relevance made after discussion. Data on study and patient characteristics, intervention and control group treatment, follow-up and outcomes and results were extracted onto a summary table.

\section{Outcomes}

We did not exclude studies on the grounds of what outcomes were reported, as the possibility existed that authors might be able to provide unpublished outcome data. However, the outcomes of interest to this review relate to pain severity.

\section{Quality assessment}

Study quality was assessed using criteria in the Cochrane risk of bias table, ${ }^{30}$ and is summarised with other study data in table 1 .

\section{Analyses}

If sufficient studies with similar outcome measures were identified, we intended to conduct an appropriate meta-analysis using Review Manager. If this was not possible, we planned a descriptive overview of studies. 


\section{Box 1 Search strategies as applied in MEDLINE}

\section{Prediction}

- risk function.mp. or risk assessment

risk equation\$.mp.

risk chart.mp.

- (risk adj3 tool\$).mp. [mp=ti, ab, ot, nm, hw, kf, px, rx, ui, sh, tn, dm, mf, dv, kw]

- risk assessment function.mp.

- risk assessor.mp.

- risk appraisal\$.mp.

- risk calculation\$.mp.

- risk calculator $\$ . m p$.

- risk factor $\$$ calculation $\$ . m p$.

- risk engine\$.mp.

- risk equation\$.mp.

- risk table\$.mp.

risk threshold\$.mp.

risk scoring method?.mp.

scoring scheme?.mp.

risk scoring system.mp.

risk prediction.mp.

predictive instrument.mp.

project\$ risk.mp.

- exp decision support techniques/

- Diagnosis, Computer-Assisted/

- Decision Support Systems, Clinical/

algorithms/

algorithm?.mp. or Algorithms/

algorythm?.mp.

decision support?.mp.

predictive model.mp.

treatment decision.mp.

scoring method\$.mp.

- (prediction\$ adj3 method\$).mp. [mp=ti, ab, ot, nm, hw, kf, $\mathrm{px}, \mathrm{rx}, \mathrm{ui}, \mathrm{sh}, \mathrm{tn}, \mathrm{dm}, \mathrm{mf}, \mathrm{dv}, \mathrm{kw}]$

- exp Risk Assessment/

- (risk? adj1 assess\$).mp. [mp=ti, ab, ot, nm, hw, kf, px, rx, ui, $\mathrm{sh}, \mathrm{tn}, \mathrm{dm}, \mathrm{mf}, \mathrm{dv}, \mathrm{kw}]$

- 1 or 2 or 3 or 4 or 5 or 6 or 7 or 8 or 9 or 10 or 11 or 12 or 13 or 14 or 15 or 16 or 17 or 18 or 19 or 20 or 21 or 22 or 23 or 24 or 25 or 26 or 27 or 28 or 29 or 30 or 31 or 32 or 33

knee.tw.

34 and 35

randomized controlled trial.pt.

controlled clinical trial.pt.

randomized.ab.

placebo.ab.

randomly.ab.

trial.ab.

groups.ab.

- (animals not (humans and animals)).sh.

37 or 38 or 39 or 40 or 41 or 42 or 43

45 not 44

36 and 46

Treatment

- Pain, Postoperative/

- ((postoperative adj6 pain $\left.{ }^{\star}\right)$ or (post-operative adj6 pain*) or post-operative-pain*).mp. [mp=title, abstract, original title, name of substance word, subject heading word, keyword heading word, protocol supplementary concept, rare disease supplementary concept, unique identifier]
- ((post-operative adj6 analgesi $\left.{ }^{\star}\right)$ or (postoperative adj6 analgesi $\left.\left.{ }^{\star}\right)\right) \cdot m p$. [mp=title, abstract, original title, name of substance word, subject heading word, keyword heading word, protocol supplementary concept, rare disease supplementary concept, unique identifier]

- ((post-surgical adj6 pain*) or (post surgical adj6 pain*) or (post-surgery adj6 pain*) or (post adj surg* adj pain*)).mp. $[\mathrm{mp}=$ title, abstract, original title, name of substance word, subject heading word, keyword heading word, protocol supplementary concept, rare disease supplementary concept, unique identifier]

- $\left(\left(\right.\right.$ post $^{\star}$ adj pain $\left.{ }^{\star}\right)$ or pain relief after or pain following surg $\left.{ }^{\star}\right)$. $\mathrm{mp}$. [mp=title, abstract, original title, name of substance word, subject heading word, keyword heading word, protocol supplementary concept, rare disease supplementary concept, unique identifier]

- ((posttreatment adj6 pain $\left.{ }^{\star}\right)$ or (pain control after adj6 surg $\left.{ }^{\star}\right)$ or ((post-extraction or postextraction or post-surg*) and (pain* or discomfort))).mp. [mp=title, abstract, original title, name of substance word, subject heading word, keyword heading word, protocol supplementary concept, rare disease supplementary concept, unique identifier]

- randomized controlled trial.pt.

- controlled clinical trial.pt.

randomized.ab.

- placebo.ab.

- randomly.ab.

trial.ab.

- 7 or 8 or 9 or 10 or 11 or 12

- 1 or 2 or 3 or 4 or 5 or 6

- 13 and 14

knee.tw.

- 15 and 16

\section{RESULTS}

Main features of the review process are summarised according to PRISMA guidelines as online supplementary material. As shown in figure 1, searches for evaluations of predictive models and treatments identified 1159 and 1886 articles, respectively.

\section{Application of predictive models}

After screening all the titles and abstracts, 16 articles were identified as potentially relevant to the review of predictive models and were evaluated in detail. Reasons for exclusion are summarised with references in the online supplementary material. No studies evaluated the effectiveness of predictive models in guiding treatment and improving long-term outcomes after knee surgery.

\section{Chronic pain management interventions}

In the review of treatments for chronic pain after knee surgery, a large majority of studies $(66 \%)$ reported analgesia or other interventions in the perioperative period. Thirty articles were judged to be potentially relevant. Reasons for exclusion of 29 studies are summarised with references in the online supplementary material.

One intervention fulfilled all inclusion criteria, and study details are summarised in table 1 . Singh et $a l^{34}$ 
evaluated the use of a botulinum toxin A injection with antinociceptive and anticholinergic activity in a randomised controlled trial. In the original randomisation, patients with simultaneous bilateral total knee replacement were included, but the published article focused on 49 patients with a unilateral replacement (or first operation in a sequential bilateral replacement). On the basis of criteria specified in the Cochrane risk of bias table, we assessed that this study was of low risk of bias though the small size of the study is a cause for concern. Patients in the trial had received a total knee replacement at least 6 months earlier and had experienced pain in their replaced knee for more than 3 months. Reduced pain intensity was apparent for the intervention compared with placebo after 2 and 3 months, although the authors suggested that meaningful pain relief was evident up to about 40 days with no increase in adverse events. No costeffectiveness analysis was performed. The authors concluded that the effect of repeated injections should be assessed in a multicentre trial, but no further study was found on inspection of the Current Controlled Trials database on 5 November 2014.

\section{DISCUSSION}

By limiting potential sources of bias, randomised controlled trials provide the best method to assess the effectiveness of healthcare interventions. Systematic reviews aim to appraise evidence from high-quality studies and can have two broad outcomes: a synthesis of knowledge to guide decision-making; or identification of deficits in the evidence base that merit further research.

The main indications for total knee replacement are pain and functional limitations caused by osteoarthritis. The widespread acknowledgement that some people will have chronic postsurgical pain after this potentially curative treatment dates largely from the introduction of patient-reported outcome measures. There is some

\begin{tabular}{|c|c|}
\hline Author & Singh et $a \beta^{\beta 4}$ \\
\hline Country & USA \\
\hline Indication & $\begin{array}{l}\text { Total knee replacement }>6 \text { months. Chronic pain }>3 \text { months ( } \geq 6 \text { points on } 10 \text {-point VAS scale). } \\
\text { Unsuccessful treatment with oral pain medication, not surgical candidate or infection identified. } \\
\text { Mean pain duration } 4.5 \text { years }\end{array}$ \\
\hline Number of patients & 49 patients with 60 total knee replacements (30 intervention: 30 control) \\
\hline Age & Mean: intervention 67.1 years; control 66.8 years \\
\hline Sex & Female: intervention $22 \%$; control $12 \%$ \\
\hline Approach & Standardised medial or lateral \\
\hline Intervention & Intra-articular injection of 100 units botulinum toxin A diluted in $5 \mathrm{~mL}$ sterile normal saline \\
\hline Control & Intra-articular injection of $5 \mathrm{~mL}$ sterile normal saline \\
\hline Follow-up interval & Up to 6 months \\
\hline \multirow[t]{11}{*}{ Outcome measures } & Proportion of responders at 2 months ( $\geq 2$ point VAS reduction) \\
\hline & Physicians' global assessment of change \\
\hline & Onset and duration of pain (20 point WOMAC pain decrease) \\
\hline & WOMAC function \\
\hline & Timed-stands test \\
\hline & Timed-up-and-go \\
\hline & Active knee flexion \\
\hline & Medical Outcomes Study Short-Form 36 (SF-36) \\
\hline & Short-form McGill Pain Questionnaire \\
\hline & Changes in analgesic medications \\
\hline & Side effects and adverse outcomes \\
\hline Economic evaluation & None reported \\
\hline Risk of bias & Overall: low \\
\hline Random sequence & Independent \\
\hline Concealment & Syringes prepared independently \\
\hline Blinding & Patients, surgeon, investigators, statistician all blind to group allocation \\
\hline Blind outcome & Assessment bind to group allocation \\
\hline Complete data & Low losses to follow-up at primary outcome intervals \\
\hline Selective reporting & Appropriate range of outcomes reported \\
\hline Other bias & None apparent \\
\hline Losses to follow-up & 2 (1:1) lost to 2 month follow-up. 7 (3:4) lost to 6 month follow-up \\
\hline Power calculation & Reported to be powered for significant improvement on WOMAC scale \\
\hline Results summary & $\begin{array}{l}\text { Pain severity reduced in } 71 \% \text { of intervention patients compared with } 35 \% \text { in placebo group at } \\
2 \text { months. Benefit also at } 3 \text { months but not at } 4 \text { months. Duration of meaningful pain relief was } 39.6 \\
(S D=50.4) \text { days in intervention group compared with } 15.7(S D=22.6) \text { days in placebo group }\end{array}$ \\
\hline
\end{tabular}



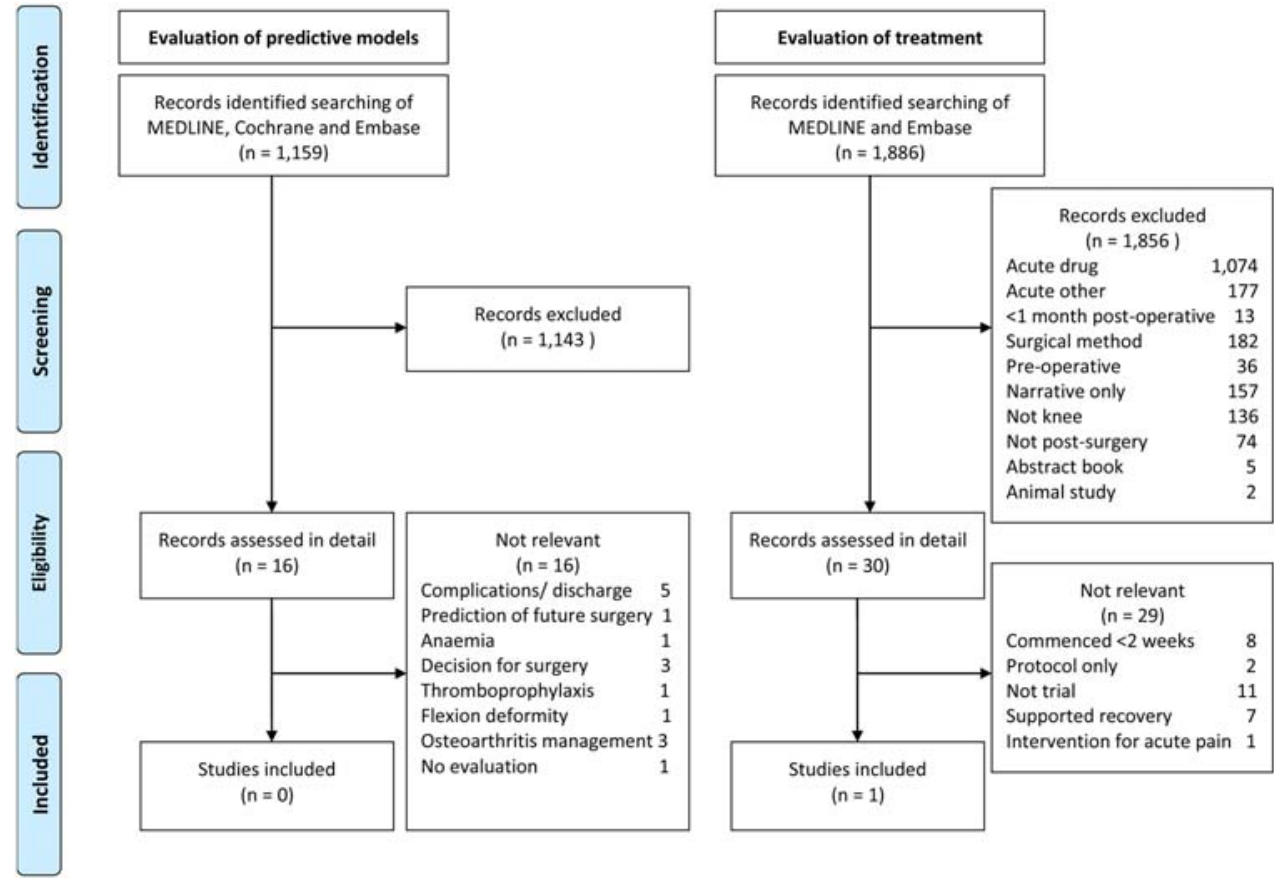

Figure 1 Systematic review flow diagram.

evidence that acute postoperative pain may impact on long-term pain, ${ }^{35}$ and a considerable number of randomised treatment evaluations have targeted reduction in acute pain with perioperative multimodal anaesthesia. ${ }^{12-15}$ Few studies have followed up patients long term, although the importance of this is now recognised. ${ }^{36}$ While acknowledging the potential importance of such methods in preventing the development of long-term pain, an appropriate body of research should also explore the issues of prediction and management. The one trial of pain management that we identified showed promise, but further research is needed to confirm the findings. Treatment of chronic pain can be challenging, and there is a need to evaluate multidisciplinary combination treatments and the benefit of matching interventions to patient characteristics. ${ }^{28} 29$

Our study might be criticised as asking research questions that are too specific and beyond the scope of randomised evaluation. However, evaluation of predictive models in guiding healthcare is recognised in other medical disciplines. For example, risk scoring has been studied in cardiovascular disease in randomised trials both as a guide for appropriate medical treatment of risk factors ${ }^{37} 38$ and lifestyle interventions. ${ }^{39}$ Without evidence that application of predictive models in total knee replacement is more effective in guiding treatment and improving outcomes than existing care, they have no value in evidence-based clinical practice.

In total knee replacement, specific biological and mechanical issues, and psychological factors relating to joint replacement should be considered in the treatment of chronic pain. The identification of one randomised trial in our review reflects an understanding that approaches to pain management after total knee replacement have features that differ from chronic pain attributable to other causes. Furthermore, a range of potential interventions with no robust evaluation were identified in our review, specifically neurostimulation, ${ }^{40-43}$ radiofrequency ablation, ${ }^{44}$ denervation, ${ }^{45}{ }^{46}$ steroid injection ${ }^{47}$ and secondary resurfacing. ${ }^{48}$ This can only be indicative of an awareness of the issue of treatment of pain after knee surgery as the literature searches were not designed to identify studies that did not report robust evaluations. While these relate specifically to orthopaedic surgery and to underlying musculoskeletal conditions, some strategies will be transferrable from more general pain management including analgesic medication, and should be considered as potential interventions in patients with long-term pain after total knee replacement.

In summary, our systematic review highlights the lack of evidence about prediction and management of chronic postsurgical pain after total knee replacement. Given the complexity of chronic postsurgical pain and the range of possible treatment options, screening and adequate referral processes are needed, so that patients can receive appropriate interventions that have the potential to improve outcomes and reduce distress. As a large number of people are affected by chronic pain after total knee replacement, the development of an evidence base about care for these patients should be a research priority.

Acknowledgements This publication is part of the STAR Project (Support and Treatment after Replacement). As such, it benefits from involvement of the whole STAR team. The STAR team includes: Dr Rachael Gooberman-Hill, Mr Andrew Beswick, Professor Ashley Blom, Mr Nick Howells, Dr Fiona MacKichan, Dr Sian Noble, Professor Tim Peters, Dr Vikki Wylde, (all at the University of Bristol); Professor Nigel Arden, Dr Andrew Judge, Professor Andrew Price (all at the University of Oxford); Mr Phil Baker (Arthritis Care); 
Ms Victoria Wells (former patient); Professor Paul Dieppe (University of Exeter); Professor Christopher Eccleston (University of Bath); Dr Julie Bruce (University of Warwick); Professor Candy McCabe (Royal National Hospital for Rheumatic Diseases); Dr Cathy Stannard (North Bristol NHS Trust).

Contributors ADB, VW and RG-H conceived and designed the study and produced the search strategy. ADB conducted the searches. ADB and VW screened abstracts and titles, assessed study inclusion and exclusion, extracted data and assessed study quality. ADB drafted the manuscript and VW and RG-H revised it critically for important intellectual content. All authors gave approval to the final manuscript.

Funding This article presents independent research funded by the National Institute for Health Research (NIHR) under its Programme Development Grants scheme (Reference Number: RP-DG-1211-10002).

\section{Competing interests None declared.}

Provenance and peer review Not commissioned; externally peer reviewed.

Data sharing statement Additional data can be accessed via the Dryad data repository at http://datadryad.org/ with the doi:10.5061/dryad.hr7k2.

Disclaimer The views expressed are those of the authors and not necessarily those of the NHS, the NIHR or the Department of Health.

Open Access This is an Open Access article distributed in accordance with the Creative Commons Attribution Non Commercial (CC BY-NC 4.0) license, which permits others to distribute, remix, adapt, build upon this work noncommercially, and license their derivative works on different terms, provided the original work is properly cited and the use is non-commercial. See: http:// creativecommons.org/licenses/by-nc/4.0/

\section{REFERENCES}

1. National Joint Registry for England and Wales: 11th Annual Report. Hemel Hempstead: NJR Centre, 2014.

2. Centers for Disease Control and Prevention. Number of all-listed procedures for discharges from short-stay hospitals, by procedure category and age: United States, 2010. Atlanta, GA: Centers for Disease Control and Prevention, 2010. www.cdc.gov/nchs/data/ nhds/4procedures/2010pro4numberprocedureage.pdf (accessed 29 Apr 2015)

3. Weinstein AM, Rome BN, Reichmann WM, et al. Estimating the burden of total knee replacement in the United States. $J$ Bone Joint Surg Am 2013;95:385-92.

4. Macrae WA. Chronic post-surgical pain: 10 years on. $\mathrm{Br} \mathrm{J}$ Anaesth 2008:101:77-86.

5. International Association for the Study of Pain. Classification of chronic pain. Descriptions of chronic pain syndromes and definitions of pain terms. Prepared by the International Association for the Study of Pain, Subcommittee on Taxonomy. Pain 1986;3:S1-226.

6. Werner MU, Kongsgaard UE. Defining persistent post-surgical pain: is an update required? Br J Anaesth 2014;113:1-4.

7. Bachmeier CJM, March LM, Cross MJ, et al. A comparison of outcomes in osteoarthritis patients undergoing total hip and knee replacement surgery. Osteoarthritis Cart 2001;9:137-46.

8. Beswick AD, Wylde V, Gooberman-Hill R, et al. What proportion of patients report long-term pain after total hip or knee replacement for osteoarthritis? A systematic review of prospective studies in unselected patients. BMJ Open 2012;2:e000435.

9. Jeffery AE, Wylde V, Blom AW, et al. "It's there and l'm stuck with it": Patients' experiences of chronic pain following total knee replacement surgery. Arthritis Care Res 2011:63:286-92.

10. Turkiewicz A, Petersson IF, Björk J, et al. Current and future impact of osteoarthritis on health care: a population-based study with projections to year 2032. Osteoarthritis Cart 2014;22:1826-32.

11. Kurtz S, Ong K, Lau E, et al. Projections of primary and revision hip and knee arthroplasty in the United States from 2005 to 2030. J Bone Joint Surg Am 2007;89:780-5.

12. Chan EY, Fransen M, Parker David A, et al. Femoral nerve blocks for acute postoperative pain after knee replacement surgery. Cochrane Database Syst Rev 2014;(5):CD009941.

13. Lin J, Zhang L, Yang H. Perioperative administration of selective cyclooxygenase-2 inhibitors for postoperative pain management in patients after total knee arthroplasty. J Arthroplasty 2013;28:207-13.

14. Marques $\mathrm{E}$, Jones $\mathrm{H}$, Elvers $\mathrm{K}$, et al. Local anaesthetic infiltration for peri-operative pain control in total hip and knee replacement: systematic review and meta-analyses of short- and long-term effectiveness. BMC Musculoskelet Disord 2014;15:220.

15. Keijsers R, van Delft R, van den Bekerom MJ, et al. Local infiltration analgesia following total knee arthroplasty: effect on post-operative pain and opioid consumption-a meta-analysis. Knee Surg Sports Traumatol Arthrosc 2013. doi:10.1007/s00167-013-2788-1 [Epub ahead of print 30 Nov 2013].

16. Lingard EA, Katz JN, Wright EA, et al. Predicting the outcome of total knee arthroplasty. J Bone Joint Surg Am 2004;86:2179-86.

17. Papakostidou I, Dailiana ZH, Papapolychroniou T, et al. Factors affecting the quality of life after total knee arthroplasties: a prospective study. BMC Musculoskelet Disord 2012;13:116.

18. Perruccio AV, Power JD, Evans HMK, et al. Multiple joint involvement in total knee replacement for osteoarthritis: effects on patient-reported outcomes. Arthritis Care Res 2012;64:838-46.

19. Sullivan $M$, Tanzer $M$, Reardon $G$, et al. The role of presurgical expectancies in predicting pain and function one year following total knee arthroplasty. Pain 2011;152:2287-93.

20. Burns LC, Ritvo SE, Ferguson MK, et al. Pain catastrophizing as a risk factor for chronic pain after total knee arthroplasty: a systematic review. J Pain Res 2015;8:21-32.

21. Vissers MM, Bussmann JB, Verhaar JAN, et al. Psychological factors affecting the outcome of total hip and knee arthroplasty: a systematic review. Semin Arthritis Rheum 2012;41:576-88.

22. Singh JA, Lewallen DG. Medical and psychological comorbidity predicts poor pain outcomes after total knee arthroplasty. Rheumatology 2013;52:916-23.

23. Brander VA, Stulberg SD, Adams AD, et al. Predicting total knee replacement pain: a prospective, observational study. Clin Orthop Relat Res 2003;416:27-36.

24. Wylde V, Dixon S, Blom AW. The role of preoperative self-efficacy in predicting outcome after total knee replacement. Musculoskeletal Care 2012;10:110-18.

25. Judge A, Arden NK, Cooper C, et al. Predictors of outcomes of total knee replacement surgery. Rheumatology 2012;51:1804-13.

26. Althaus A, Hinrichs-Rocker A, Chapman R, et al. Development of a risk index for the prediction of chronic post-surgical pain. Eur J Pain 2012;16:901-10.

27. Wyatt JC, Altman DG. Commentary: prognostic models: clinically useful or quickly forgotten? BMJ 1995;311:1539-41.

28. Turk DC, Wilson HD, Cahana A. Treatment of chronic non-cancer pain. Lancet 2011:377:2226-35.

29. Merle $\mathrm{C}$, Brendle $\mathrm{S}$, Wang $\mathrm{HL}$, et al. Multidisciplinary treatment in patients with persistent pain following total hip and knee arthroplasty. $J$ Arthroplasty 2014;29:28-32.

30. Higgins JPT, Green S, eds. Cochrane handbook for systematic reviews of interventions Version 5.1.0 [Updated March 2011]. The Cochrane Collaboration, 2011. http://www.cochrane-handbook.org

31. Moher D, Liberati A, Tetzlaff J, et al. Preferred reporting items for systematic reviews and meta-analyses: the PRISMA statement. J Clin Epidemiol 2009;62:1006-12.

32. Chaparro Luis E, Smith Shane A, Moore RA, et al. Pharmacotherapy for the prevention of chronic pain after surgery in adults. Cochrane Database Syst Rev 2013;(7):CD008307. http:// onlinelibrary.wiley.com/doi/10.1002/14651858.CD008307.pub2/ abstract

33. Brindle P, Beswick A, Fahey $\mathrm{T}$, et al. Accuracy and impact of risk assessment in the primary prevention of cardiovascular disease: a systematic review. Heart 2006;92:1752-9.

34. Singh JA, Mahowald ML, Noorbaloochi S. Intraarticular botulinum toxin A for refractory painful total knee arthroplasty: a randomized controlled trial. J Rheumatol 2010;37:2377-86.

35. Kehlet $\mathrm{H}$, Jensen TS, Woolf CJ. Persistent postsurgical pain: risk factors and prevention. Lancet 2006;367:1618-25.

36. Wylde V, Lenguerrand E, Gooberman-Hill R, et al. The effect of local anaesthetic infiltration on chronic post-surgical pain after total hip and knee replacement: the APEX randomised controlled trials. Pain 2015. doi:10.1097/j.pain.0000000000000114 [Epub ahead of print 5 Feb 2015].

37. Beswick A, Brindle P. Risk scoring in the assessment of cardiovascular risk. Curr Opin Lipidol 2006;17:375-86.

38. Vagholkar S, Zwar N, Jayasinghe UW, et al. Influence of cardiovascular absolute risk assessment on prescribing of antihypertensive and lipid-lowering medications: a cluster randomized controlled trial. Am Heart J 2014;167:28-35.

39. Willis A, Davies M, Yates T, et al. Primary prevention of cardiovascular disease using validated risk scores: a systematic review. J R Soc Med 2012;105:348-56.

40. Billet B, Hanssens K, Nagels W, et al. Treatment of post-surgical knee pain with dorsal root ganglion (DRG) neurostimulation (Abstract). Pain Practice 2014;14:112. 
41. Bouche B, Eisenberg E, Karmakar MK, et al. Facilitation of diagnostic and percutaneous trial lead placement with ultrasound guidance for peripheral subcutaneous field stimulation on infrapatellar branches of saphenous nerve (Abstract). Reg Anesth Pain Med 2011;2:E158.

42. Lowry AM, Simopoulos TT, Lowry AM, et al. Spinal cord stimulation for the treatment of chronic knee pain following total knee replacement. Pain Physician 2010;13:251-6.

43. Koulousakis A. Peripheral subcutaneous nerve stimulation (PNS) in neuropathic pain: seven years of experience. Neuromodulation 2013;16:e153.

44. Drakes S, Kim SY, Wahezi SE, et al. Ultrasound guided radiofrequency ablation of the saphenous nerve for treatment of saphenous neuropathy: a case report (Abstract). PM\&R2013;1:S306.
45. Dellon AL, Mont MA, Krackow KA, et al. Partial denervation for persistent neuroma pain after total knee arthroplasty. Clin Orthop Relat Res 1995;316:145-50.

46. Nahabedian MY, Mont MA, Hungerford DS. Selective denervation of the knee: experience, case reports, and technical notes. Am J Knee Surg 1998;11:175-80.

47. Chaiyakit $\mathrm{P}$, Meknavin S, Pakawattana V, et al. Results of peri-articular steroid injection in the treatment of chronic extra-articular pain after total knee arthroplasty. J Med Assoc Thai 2012;95(Suppl 10): S48-52.

48. Muoneke HE, Khan AM, Giannikas KA, et al. Secondary resurfacing of the patella for persistent anterior knee pain after primary knee arthroplasty. J Bone Joint Surg Br 2003;85:675-8. 